\section{Using Artificial Intelligence to improve prediction and prevention of violence}

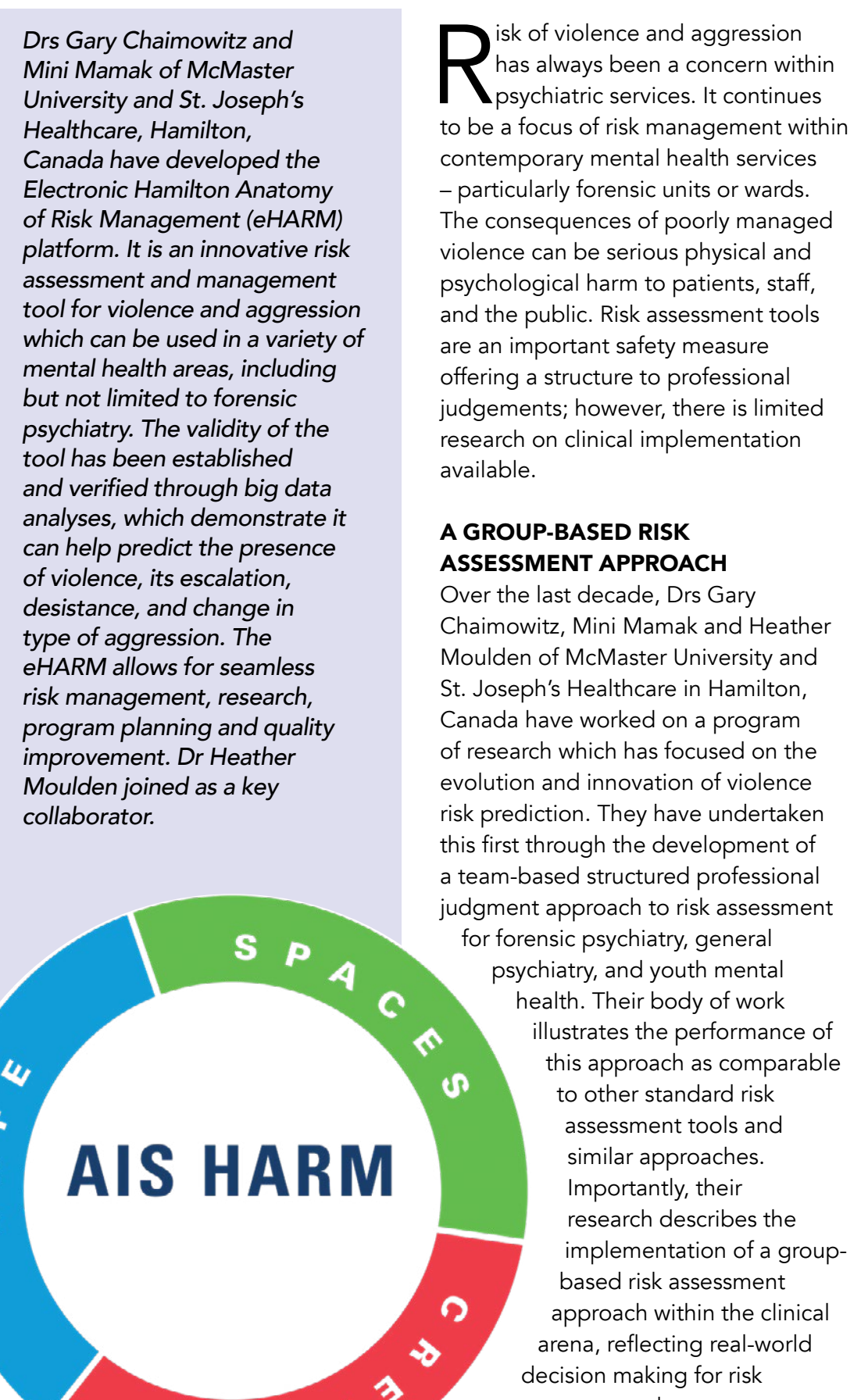

$\Rightarrow \quad$ decision making for risk
This risk assessment approach has recently been subjected to machine learning modelling to further strengthen and improve predictior negative outcomes.

\section{MACHINE LEARNING}

\section{AND BIG DATA}

ted machine learning fist test the efficacy of this approach in the risk assessment arena, with an eye to ultimately increase the predictive power of their risk assessment can predict violence within groups with some certainty, they are unable to make individual predictions for a given patient. Machine learning is a branch of artificial intelligence: a computer learns and adapts to new data without human intervention. This provided the research team with the capacity to statistically nalyse the big data required. Given that machine learning is untethered by some of the conventions or assumptions this approach may also allow for the identification of novel risk factor candidates, which perhaps had not be previously unearthed. Furthermore, machine learning modelling can increase precision over time to offer individualized predictions.

Dr Chaimowitz, Dr Mamak and Dr Moulden used this machine learning method to process the large amount of information from a representative and diverse sample of patients in forensic mental health settings. Based on this, they developed and evaluated a risk assessment and management tool, the Electronic Hamillon Anatomy of Risk tool. While other risk assessment tools Management (eHARM) plattorm. Clinica,

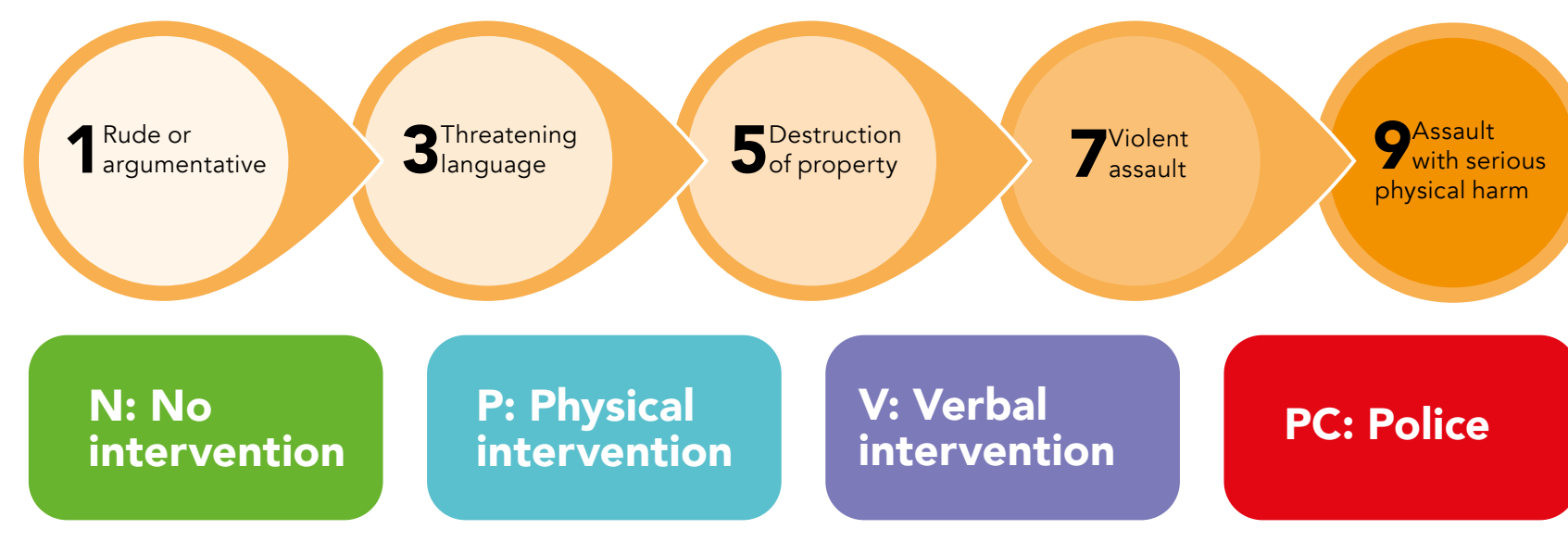

Figure 1. The AIS method consists of a two-part code: a number from 1-9 which describes the level of aggression experienced by the patient and a

were considered as potentia pedictors W of violence and separate models were Their Their results suggest that their machine. previous gold-standard risk assessment tools. Unlike these existing tools, patient-focused eHARM (as opposed data validity of the other two versions: youth and correctional. These tools part code, initially a number from 1

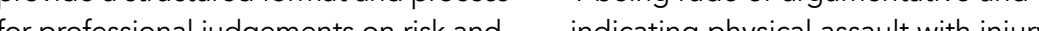
management of violence.

\section{AlS is a method for describing and} indicating physical assault with injury. leter referring to the staff intervention: $N, P, V$ and $P C, N$ is no intervention, $P$ is physical intervention, $V$ is verba eHARM) allows Unlike existing tools, eHARM allows PC refers to police for the prediction unlike existing tools, eHARM allows simple and management for the prediction of violence at an coding facilitates staff individual level,

and specifically for clinicians dealing with immediate issues of risk management. individual level. and management
with a common

THE RISK ASSESSMENT AND MANAGEMENT TOOL The risk assessment and management tool developed by the team has Incidents Scale (AIS) the Electronic Hamilton Anatomy of Risk Manago HARM) and the OHARM aggrogat: From their initial eHARM for forensic psychiatry, they developed four versio the forensic version, the general. version, the youth version, and the correctional version.

The forensic version was developed first, later improved using big data and machine learning. The big data developed from its implementation facilitated even bigger data sets. Further studies were undertaken to develop the general version with input from clinician and with data collected through mplementation. This will lead to big similar approach was used to gain big common style of language to provide a cumulative and graphic picture of a patient's record of aggression. This is to facilitate interpretation of the leve of aggression experienced by the patient and any patterns, including

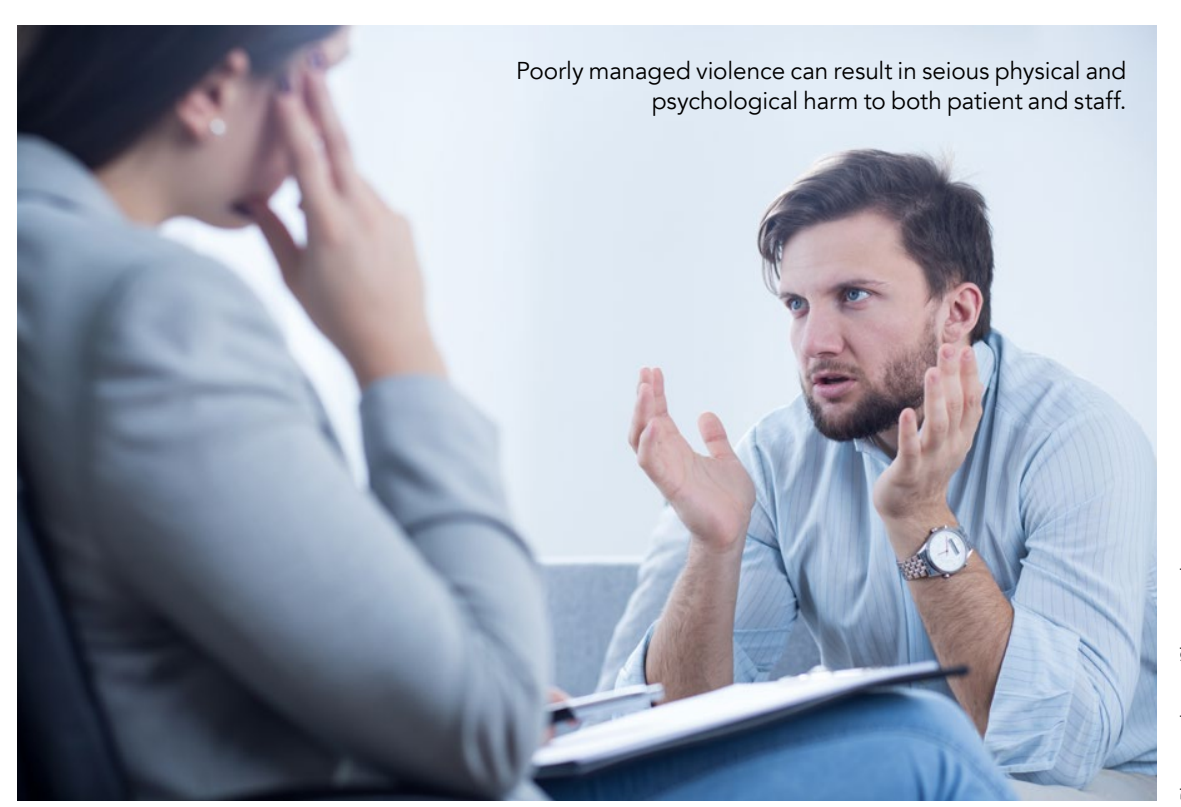

accessible language. It also provides a concise and simple method for documentation.

The four versions of the Electronic 

and short-term risk. Many traditional these discussions and facilitates easy facilitate research analyses at a group clinical assessment tools to support decisions regarding risk of violence in historic (such as previous violent behaviour) static (stable factors that do not change over time) and dynamic, changeable factors to predict immin risk assessment approaches rely on historical or static factors, which can be time consuming to complete, but offer little information about more imminent violence or ways to intervene.

a documentation process that captures This understing is the basis for the formulation of risk manageme strategies and forms the individualised patient report.

The eHARM aggregator is a separate component within the eHARM where individual reports can be downloaded as non-identifiable data. This can

variety of settings, such as inpatient o vitpatient and group homes.

\section{CONCLUSION} . Its three components - AIS, eHARM and eHARM aggregator - and three fluent stages - past, present and future - offer a wide scope of assessment and implementation for the management of violent behaviour. The four versions of

dynamic risk

assessments

perform better

for short-term

predictions of

identify points of intervention.

AIS offers the team a clear simple eHARM allow for a language in which to discuss incidents arenas in which it and ongoing care needs. can be used. level which enlightens the establishment of trends in behavioural patterns and allows for cross system research, qually improvement and service planning with no additional effort. formation available in assessing, ostering, and facilitating the cocumentation of team members' management process. There are three fluent stages to the assessment: Past, Current, and Future. The assessor/s move from exploring the past historica factors to what is happening currently for the patient to arrive at an estimate of future risk of violence. This is similar to other risk assessments; however, HARM works through a multiprofessional team-based approach Als offers the team a clear simple language in which to discuss incidents in psychiatry, with its team-based approach, and the recent application to big data risk assessment processes. The Aggressive Incident Scale is an easy-to-use scale to measure aggressive incidents combined with the patient leve eHARM and the eHARM aggregator. Dr Chaimowitz, Dr Mamak and Dr Mould have developed a rigorous suite of risk assessment and management tools. The tools can be used within a variety psychiaty to forensic revices and in a
The eHARM platform proves to be a continuous risk assessment and

eHARM is a ground-breaking innovation The use of big
data through with the empircal research to provide with the empirical research to provide simple language facilitates robust team based clinical practice. This research has also proved useful in understanding reoffence scenarios and age-related violence through the development of the youth and correctional versions. Using this tool, a mental health professional can more accurately predict the presence of violence, escalation, desistance, and changes in the type of violence of individuals as well as groups, and formulate appropriate risk prevent future aggression

The eHARM allows for seamless risk management, research, program planning, and quality improvement.

\section{The researchers used a machine learning approach
to risk assessment for forensic psychiatry, general
psychiatry, and youth mental health.}

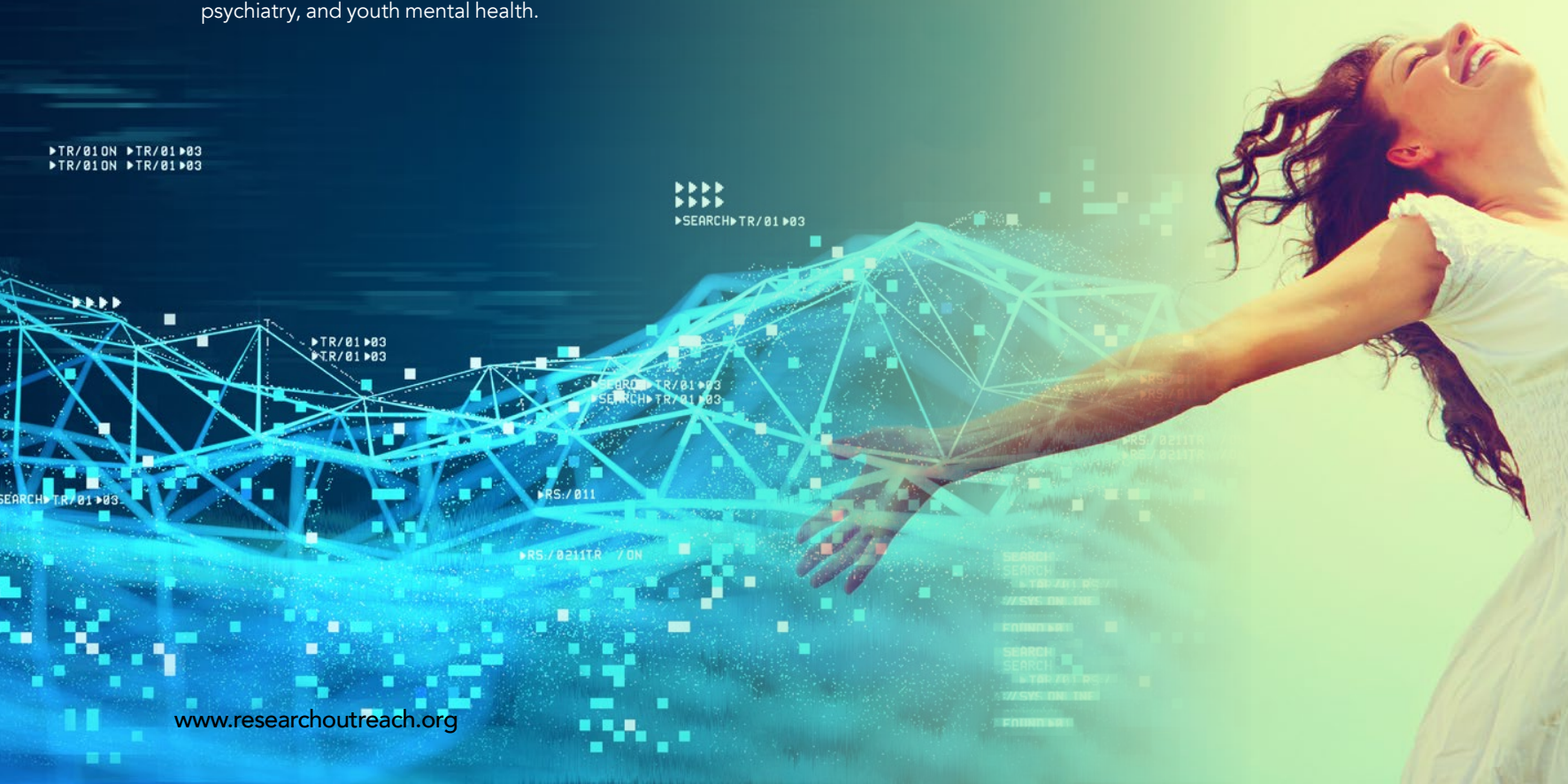

\section{Behind the Research}

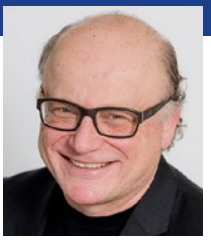

\section{Gary \\ Chaimowitz}

A

Mini

Mamak

: gchaimow@stjoes.ca E: chaimow@mcmaster.ca T: +1 905-522-1155 ext. 35424 Fax: +1 9053815606 W.htps.//psychiatry.mcmaster.ca/home FPl: https://Www.forensicpsychiatryinstitute.com/ IJRR: https://mulpress.mcmaster.ca//j] R\&R: https://www.riskandrecoveryconference.com/ HARM: $h$ ttps://www.ais-harm.com/ @ @ForensicPsychln
Forensic Psychiatry Program: https:///bit.ly/2T79.JKA International Forensic Psychiatry Lecture Series: https://bit.ly/2TRGRB

\section{Research Objectives}

The research team have developed the Electronic Hamilton innovative risk assessment and management tool which can

be used in a variety of mental health areas.

\section{Detail}

Address

Dr Gary Chaimowitz

. Joseph's Healthcare Hsychiatry Program

100 West $^{\text {th }}$ Street, Hamilton, ON L8N 3K7

Bio

Gary Chaimowitz is a Professor in the Department of

University in Hamilton. He is the Head of Service, Forensic

Unive

Currently a member of the Ontario and Nunavut Review

Boards, Dr Chaimowitz is a member of the Forensic

College of Physician and Surgeons of Canada.

Mni Mamak is the Senior Psychologist in the Forensic

Psychiatry Program at St. Joseph's Healthcare Hamilton.
She is an Associate Clinical Professor with the Departmen

Psychiatry and Behavioural Neurosciences. Her research

assessment Dr Mamak works with various police services

training and education

Heather Moulden is a clinical forensic psychologist at St.

Joseph's Healthcare, an Associate Clinical Professor with the

and Associate Member in the Department tof Psychology,

Neuroscience and Behaviour at McMaster University. Her

research and clinical interests include problematic sexual

behaviour, enhancing forensic reha

Funding

- Janssen provided seed funding

Collaborators
- Devon Watts Casey Upfold

- Andrew Olagunju - Luiza Gea

- Eartlyn Mullally $\quad$ : Sylan Gatner

\section{References}

Chaimowitz, G.A., Mamak, M. (2018). AIS - HARM. [online]. Available at: https://www.ais-harm.com/ [Accessed 06/05/2

Chaimowitz, G.A., Mamak, M., Moulden, H.M., Furimsky,

.., Olagunju, A.T. (2020). Implementation of risk assessment tools in psychiatric services. American Society for Healthcare org/10.1002/hrm.21405

Cook, A.N., Moulden, H.M., Mamak, M., Lalani, S., Messina, K., Chaimowitz, G. (2018). Validating the Hamilton Anatomy of Risk Management-Forensic Version and the Aggressive https://doi.org/10.1177/1073191116653828

Gatner, D.T., Moulden, H.M., Mamak, M., Chaimowitz, G.A. (2021). At Risk of What? Understanding Forensic Psychiatric Inpatient Aggression through a Violence Risk Scenario Planning Lens. International Journal of Forensic Mental Health.
Available at: httrs://doi.org/10.1080/14999013 2021.1899343 Watts, D., Moulden, H., Mamak, M., Upfold, C., Chaimowitz, with psychiatric disorders - A machine learning approach Journal of Psychiatric Research, 138, 146-154. Available at https://doi.org/10.1016/j.jpsychires.2021.03.026

\section{Personal Response}

What inspired you to conduct this research?

I Violence risk assessment and management

has progressed significantly over the years but had

plateau'd at the level of structured professional

judgment tools. We saw an opportunity to advance the and management, as well utilising the advances that big and eHARM Aggregator was the result.

\begin{tabular}{|l|l|l|l|l|}
\hline rensic & McMaster International \\
Schiatry
\end{tabular}

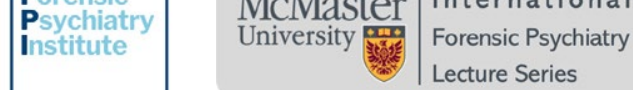

